\title{
Role of Stromal Cell-Derived Factor-1 in Patients With Non-ST Elevation Acute Coronary Syndrome
}

\author{
Guoxin Tong, ${ }^{1,2}$ MSc, Ningfu WANG, ${ }^{2}$ MD, Yujie ZHOU, ${ }^{1}$ MD, Jianhang LenG, ${ }^{3}$ MD, \\ Wei GaO, ${ }^{2}$ MSc, Xiaoshan Tong, ${ }^{2}$ MSc, Yun Shen, ${ }^{2}$ MD, Jianmin YANG, ${ }^{2}$ MD, \\ Xianhua Ye, ${ }^{2} \mathrm{MD}$, Liang Z $\mathrm{HOU},{ }^{2} \mathrm{MD}$, and $\mathrm{Yan}_{\mathrm{GAO}}{ }^{2} \mathrm{MD}$
}

\begin{abstract}
SUMMARY
The predictive value of stromal cell-derived factor-1 (SDF-1) has not been established in patients with non-ST elevation acute coronary syndrome (non-STEACS). A total of 678 consecutive patients with non-STEACS and moderate to high TIMI (Thrombolysis In Myocardial Infarction) risk scores were recruited. All patients underwent an early invasive strategy and then were followed-up for 18 months for clinical events. Left ventricular remodeling was assessed by echocardiography. Plasma concentrations of SDF-1 and N-terminal pro-B-type natriuretic peptide (NT-proBNP) were analyzed. SDF-1 level was an independent predictor of left ventricular remodeling $(\mathrm{OR}=2.95,95 \% \mathrm{CI}=2.02-4.30, P<$ 0.001). Cox regression analysis demonstrated that both SDF-1 and NT-proBNP levels were significant independent predictors of death, myocardial infarction, or heart failure $(\mathrm{HR}=2.45,95 \% \mathrm{CI}=1.71-3.50, P<0.001 ; \mathrm{HR}=3.71,95 \% \mathrm{CI}$ $=2.41-5.70, P<0.001$, respectively). The area under the ROC curves for SDF-1 (0.776) and NT-proBNP $(0.817)$ were similar. The logistic model with both markers yielded a larger area under the ROC curve $(0.862)$ than that of SDF-1 $(P<$ $0.001)$ or NT-proBNP $(P=0.0001)$ alone. In patients stratified by NT-proBNP (above $615.4 \mathrm{pmol} / \mathrm{L}), \mathrm{SDF}-1$ (above $2175.1 \mathrm{pg} / \mathrm{mL})$ was associated with poorer outcome $(P<0.001)$. Findings were similar for death and heart failure as individual endpoints. In non-STEACS, higher SDF-1 levels were a significant predictor of death, myocardial infarction, or heart failure independently of baseline clinical characteristics and NT-proBNP, and the combination of SDF-1 and NTproBNP significantly improved risk stratification. These data highlight the prognostic value of multiple, complementary biomarkers in non-ST elevation acute coronary syndrome. (Int Heart J 2014; 55: 219-227)
\end{abstract}

Key words: Natriuretic peptide, Ventricular remodeling, Prognosis

$\mathrm{R}$ ecent improvements in medical therapy and the management of acute coronary syndrome (ACS) have impacted the incidence and extent of postischemic left ventricular (LV) remodeling, including reperfusion therapy, ${ }^{1)}$ angiotensin-converting enzyme inhibitors, ${ }^{2)}$ and beta-blockers. ${ }^{3)}$ However, despite contemporary treatment, it was reported that myocardial remodeling is still a common outcome in patients with ACS. ${ }^{4}$ It is important to consider that adequate reperfusion in patients with ACS salvages myocardium and reduces mortality. However, successful restoration of epicardial coronary artery patency does not always lead to adequate reperfusion at the microvascular level. ${ }^{5)}$ Hence, LV remodeling and subsequent heart failure (HF) remain a leading cause of morbidity and mortality after ACS. Clinical features may be useful for screening patients who are at risk of developing such complications after ACS, but they lack sensitivity and specificity. Biomarkers are emerging as a useful tool for predicting prognosis in such patients. B-type natriuretic peptide and its more stable counterpart, $\mathrm{N}$-terminal pro-B-type natriuretic peptide (NT-proBNP), have shown great promise in this area, ${ }^{6}$ covering a range of acute coronary syndromes. ${ }^{7}$ At the same time, the emerging new peptides may also become clinically useful in the future. Stromal cell-derived factor-1 (SDF-1, also known as CXCL12), a constitutively expressed chemokine, has attracted much attention in the treatment of ischemic diseases. SDF-1 expression is instantly increased in the heart after myocardial infarction (MI). ${ }^{8)}$

Experiments have demonstrated that enhanced SDF-1 after myocardial ischemia results in the migration of stem cells into injured heart which leads to increased vascular density, enhanced tissue regeneration, and improved cardiac function. ${ }^{9}$ In addition, administration of SDF-1 promotes myocyte survival in the heart 72 hours after MI. ${ }^{10)}$ It was reported that SDF induces borderzone neovasculogenesis, attenuates adverse ventricular remodeling, and preserves ventricular function after myocardial infarction in rats. ${ }^{11)}$

From the ${ }^{1}$ Department of Cardiology, Beijing Anzhen Hospital, Capital Medical University, Beijing, Departments of ${ }^{2}$ Cardiology and ${ }^{3}$ Clinical Laboratory Medicine, Hangzhou Hospital (Hangzhou No.1 Municipal Hospital), Nanjing Medical University, Hangzhou, China.

This work was supported by a Project Grant from the Institute of Health of Zhejiang Province of China (No. 2007A157), and a Grant from Hangzhou Municipal Science and Technology Bureau Foundation (No. 20100733Q03).

Address for correspondence: Yujie Zhou, MD, Department of Cardiology, Beijing Anzhen Hospital, Capital Medical University, No. 2, Anzhen Road, Chaoyang District, Beijing 100029, China. E-mail: sunnystewen@ sina.com.

Received for publication September 28, 2013. Revised and accepted November 12, 2013

Released advance online J-STAGE May 7, 2014.

All rights are reserved to the International Heart Journal Association. 
Although the effects of SDF-1 have been extensively studied in vitro and in animal models, the role of SDF-1 in humans, especially in the left ventricular remodeling and prognostication of non-STEACS patients, is still unknown. In the present study, we investigated whether SDF-1 would be of benefit in determining the prognosis after non-STEACS, particularly for death, MI and HF. SDF-1 was also compared with NT-proBNP, a peptide with established prognostic value in non-STEACS patients, to determine the efficacy of SDF-1. ${ }^{12)}$

\section{MeTHODS}

Study population: The study group consisted of 678 patients with non-STEACS who were admitted to our centers from June 2009 to May 2011. Eligible patients were $\geq 40$ years of age, with $\geq 1$ episodes of angina that lasted $\geq 5$ minutes within 24 hours of admission and either a positive cardiac troponin test or $\geq 0.1 \mathrm{mV}$ of ST-segment depression or T-wave inversion $\geq 0.1 \mathrm{mV}$. The TIMI (Thrombolysis In Myocardial Infarction) risk score was calculated and patients with a risk score $\geq 3$ were included in the study. The TIMI UA/NSTEMI risk score (range, 0-7), which was derived from the TIMI 11B clinical trial and consists of 7 equally-weighted and dichotomous variables, ${ }^{13)}$ was also calculated. Exclusion criteria were active infection, neoplastic disease, loss of consciousness, pregnancy, chronic renal disease (creatinine $>150 \mu \mathrm{mol} / \mathrm{L}$ ), degenerative or rheumatic valvular disease, prior valvular or any other prosthetic material or intracardiac defibrillator replacement, and use of dipeptidylpeptidase (DPP4) inhibitors.

All patients underwent an early invasive strategy within 36 hours of admission on the basis of standard medical treatment. Coronary angiography was performed according to the Judkins technique, and images of the coronary tree were obtained in routine, standardized projections in all patients. A significant stenosis was considered if $\mathrm{a} \geq 50 \%$ diameter reduction in the main coronary artery or in one of its branches was present. Revascularization was recommended in patients with $\mathrm{a} \geq 70 \%$ diameter stenosis in any artery that supplied a substantial proportion of the myocardium. According to the coronary anatomy, angioplasty and coronary stenting were chosen as the primary revascularization strategies.

In this study, 227 age- and gender-matched control subjects were selected from individuals who received medical check in our hospitals and the following inclusion criteria were used: no history of angina or other atherosclerotic vascular diseases, and normal exercise electrocardiogram stress testing. The estimated glomerular filtration rate (eGFR) of these subjects was calculated from the simplified formula derived from the Modification of Diet in Renal Disease (MDRD) study, previously validated in patients with $\mathrm{HF}^{14)}$ Written informed consent was obtained from all enrolled participants and this study was approved by the local Ethics Committee.

Echocardiographic measurements: Two-dimensional echocardiograms with Doppler, performed with the same scanner for each patient, were recorded at baseline and at 6 months. The videotapes were evaluated in a blinded fashion. The echocardiograms were analyzed with the use of a dedicated offline echocardiography analysis system (Vingmed Vivid Five Imaging Systems, GE healthcare, USA). LV end-systolic volume (LVESV), LV end-diastolic volume (LVEDV), and LVEF were measured by Simpson's method in the apical 4-chamber view, which was used for the main analyses, as well as the apical 2-chamber view when possible. LV mass was calculated from the 2-dimensional parasternal long-axis view with the use of the Penn cubed formula. ${ }^{15)}$ LVESV index (LVESVI), LVEDVI, and LV mass index (LVMI) were determined by dividing volume or mass by body surface area. In addition, 20 patients were randomly selected for evaluation of the interobserver and intraobserver variability of LV mass measurement by 2 independent observers. Mean percent error was calculated as the absolute difference divided by the average of the two observations. Intraobserver and interobserver LV mass variabilities were $<6 \%$ and $<10 \%$, respectively.

Postischemic left ventricular remodeling was defined as a $20 \%$ increase of left ventricular end-diastolic and end-systolic volumes at the end of follow-up (6 months) compared with baseline measurements. ${ }^{16)}$

Blood sampling and laboratory investigations: Venous blood was withdrawn from the antecubital vein into EDTA tubes at admission. After samples were centrifuged at $2500 \mathrm{rpm}$ for 10 minutes, aliquots of the samples were stored at $-80^{\circ} \mathrm{C}$ until analyzed in a single batch. Plasma SDF-1 concentrations were measured by duplicate determination using a commercially available ELISA method (Quantikine, R\&D Systems). The lower limits of detection were $18 \mathrm{pg} / \mathrm{mL}$ for SDF-1. The mean intra-assay and inter-assay coefficients of variance were all less than $4.5 \%$. Plasma NT-proBNP levels were quantified with an electrochemiluminescence immunoassay and an Elecsys 2010 (Roche Diagnostics, Mannheim, Germany). The analytical sensitivity was $0.6 \mathrm{pmol} / \mathrm{L}$ with a measuring range between 0.6 and 11,600 pmol/L. Within and between assays, coefficients of variation were acceptable at $2.3 \%$ and $4.8 \%$, respectively. The assay is unaffected by bilirubin, haemolysis or lipaemia with no stated cross-reactions with atrial natriuretic peptide (ANP), $\mathrm{N}$-terminal-proANP, BNP, C-type natriuretic peptide, aldosterone, angiotensin (I, II, III), or renin. The levels of serum highsensitivity C-reactive protein (hs-CRP) were measured by immunonephelometry using the $\mathrm{BN}^{\mathrm{TM}}$ system (Dade Behring Inc, Newark, DE, USA; lower detection limit $<0.15 \mathrm{mg} / \mathrm{L}$ ). Cardiac troponin I was measured with the Opus Troponin I assay (Behring Diagnostics, Westwood, MA, USA) performed on an Opus Plus analyser, with a detection limit of $0.4 \mathrm{ng} / \mathrm{L}$. All other biochemistry measurements were carried out by the biochemistry department of our institution using standard methods.

Follow-up and definition of endpoints: The patients were followed up for events occurring during the next 18 months, using a standardized protocol that included outpatient visits, telephone contacts, and the recording of recurrent cardiac events.

The primary endpoints were the composite occurrence of major adverse clinical events, including death (all-caused death), recurrent myocardial infarction (MI) (nonfatal), or readmission due to advanced heart failure (HF) (defined as a hospital readmission for which heart failure was the primary reason). We also investigated death, recurrent MI, and advanced $\mathrm{HF}$ as individual secondary endpoints. Recurrent myocardial infarction was defined by the presence of recurrent ischemic symptoms or electrocardiographic changes accompanied by a creatine kinase concentration that was more than twice the upper limit of the normal range or more than $50 \%$ higher than the previous value obtained during hospitalization. 
Table I. Characteristics of Control Subjects and Non-STEACS Patients

\begin{tabular}{lccr}
\hline & Control Subjects & Non-STEACS Patients & $P$ \\
\hline$n$ & 227 & 678 & 0.658 \\
Age, years & $66(62-74)$ & $361(53.2)$ & 0.292 \\
Male, $n(\%)$ & $130(57.3)$ & $23.6 \pm 3.1$ & $<0.001$ \\
BMI, $\mathrm{kg} \cdot \mathrm{m}^{-2}$ & $22.4 \pm 4.1$ & $247(36.4)$ & 0.747 \\
Current smoking, $n(\%)$ & $80(35.2)$ & $365(53.8)$ & 0.345 \\
Hypertension, $n(\%)$ & $114(50.2)$ & $139(20.5)$ & 0.166 \\
Diabetes mellitus, $n(\%)$ & $37(16.3)$ & $230(33.9)$ & 0.328 \\
Hypercholesterolemia, $n(\%)$ & $69(30.4)$ & $67.1(50.8-87.3)$ & 0.007 \\
eGFR, mL $\cdot$ min $^{-1} \cdot 1.73 \mathrm{~m}^{-2}$ surface area & $74.3(55.7-94.1)$ & $2041.8(1845.8-2247.5)$ & $<0.001$ \\
SDF-1, pg/mL & $1508.8(1102.8-2016.3)$ & $615.4(391.8-948.7)$ & $<0.001$ \\
NT-proBNP, pmol/L & $91.9(51.8-273.9)$ & & \\
\hline
\end{tabular}

Data are shown as number (percentage) or mean \pm SD or median (25th to 75th percentiles). BMI indicates body mass index; Current smoking, defined as at least 1 cigarette per day for at least 1 year; Hypercholesterolemia, defined as using cholesterol-lowering medicines or serum total cholesterol level $\geq 200 \mathrm{mg} / \mathrm{dL}$; eGFR, estimated glomerular filtration rate; SDF-1, stromal derived factor-1; and NT-proBNP, N-terminal pro-B-type natriuretic peptide.

Advanced heart failure requiring hospitalization was diagnosed by clinical signs and symptoms, and chest radiographic findings.

Statistical analysis: Statistical analyses were performed on SPSS version 16 (SPSS, Inc., Chicago, Ill., USA). Data are expressed as the mean \pm standard deviation (SD) for normally distributed variables and median (25th to 75th percentiles) for non-normally distributed variables. Qualitative data are presented as numbers (percentages). If the variables were normally distributed, the comparison was performed using the MannWhitney $U$ test. Otherwise, the comparison was evaluated by $t$ test. For categorical variables, chi-square tests were performed. Correlations between plasma SDF-1 and other biochemistry indexes were assessed using Spearman's rank correlations test. To test the independent predictive power for LV remodeling, binary logistic regression analyses were conducted. Logistic regression models were constructed with the main covariates (age, male, BMI, hypertension, diabetes mellitus, prior MI, peak troponin I, use of ACE inhibitor or ARB, use of $\beta$-blocker, SDF-1, NT-proBNP, hs-CRP). To test the independent predictive power for death, MI or HF of peptide levels, survival analyses with Cox proportional hazards modeling and KaplanMeier models were conducted. Cox models were constructed with the following variables entered simultaneously, these variables included age; male; BMI; current smoking; past medical history of hypertension, diabetes mellitus, hypercholesterolemia, MI, chronic HF, or revascularization; ST-depression $\geq 0.1$ $\mathrm{mV}$; peak troponin I; eGFR; delay time (defined as the time from symptom onset to admission); admission to balloon time (defined as the time from admission to the first balloon inflation); Killip class $\geq$ II; left main artery disease; triple vessel disease; SDF-1; NT-proBNP; hs-CRP; therapy with angiotensin-converting enzyme inhibitor (ACEI) or angiotensin receptor blocker (ARB), $\beta$-blocker, statin, aspirin, clopidogrel, and tirofiban. In addition, receiver operating characteristic (ROC) analysis was performed and the area under ROC curve was calculated. Comparisons between two ROC curves were conducted by the method of Hanley and McNeil. ${ }^{17)}$ A $P$ value of $<0.05$ was considered statistically significant.

\section{Results}

Baseline characteristics of control subjects and non-STEACS patients: Table I summarizes the baselines characteristics of the control subjects and non-STEACS patients. Compared with the control subjects, the patients with non-STEACS tended to have a higher BMI and lower eGFR. No significant differences in the other baseline clinical data were observed between the control group and non-STEACS group. The concentrations of SDF-1 and NT-proBNP were significantly higher in non-STEACS patients than those in the control subjects. The median level of SDF-1 was 1,508.8 pg/mL in control subjects in our study, which was higher than the $1,264 \mathrm{pg} / \mathrm{mL}$ of healthy volunteers in a previous study. ${ }^{18)}$ There were some cardiovascular risk factors in control subjects in our study, such as current smoking, hypertension, diabetes mellitus, and hypercholesterolemia, which are known to play a role in vascular injury. It has been reported that the expression of SDF-1 is increased in injured arteries. ${ }^{19)}$ In the control subjects with no cardiovascular disease, NT-proBNP was significantly correlated with eGFR and age $(r=-0.445, P<0.001 ; r=0.335, P<$ 0.001 , respectively). SDF-1 was also significantly correlated with age $(r=-0.098, P=0.033)$, but not with eGFR $(r=0.059$, $P=0.375)$.

Baseline characteristics for non-STEACS patient strata according to the levels of SDF-1: The cutoff point of SDF-1 was identified by the receiver operating characteristic curves. The value was $2,175.1 \mathrm{pg} / \mathrm{mL}$ and the area under the curve was 0.776 . The sensitivity and specificity of the composite MACE predicted by the critical value was $72.5 \%$ and $83.2 \%$, respectively (Figure 1). Patients were classified into 2 groups: (1) lower SDF-1 group $(<2,175.1 \mathrm{pg} / \mathrm{mL})(n=444)$ and $(2)$ higher SDF-1 group $(>2,175.1 \mathrm{pg} / \mathrm{mL})(n=234)$. The baseline clinical characteristics are presented in Table II. There was no significant difference in gender; BMI; hypercholesterolemia; eGFR; admission to balloon time; and use of statin, aspirin, and clopidogrel between the 2 groups. However, compared with the lower SDF-1 group, the higher SDF-1 group had significantly greater incidences of current smoking, hypertension, diabetes mellitus, prior MI, prior $\mathrm{CHF}$, prior revascularization, ST-depression $\geq 0.1 \mathrm{mV}$, Killip class $\geq$ II, left main artery disease, and triple vessel disease; less use of $\beta$-blockers, ACE in- 


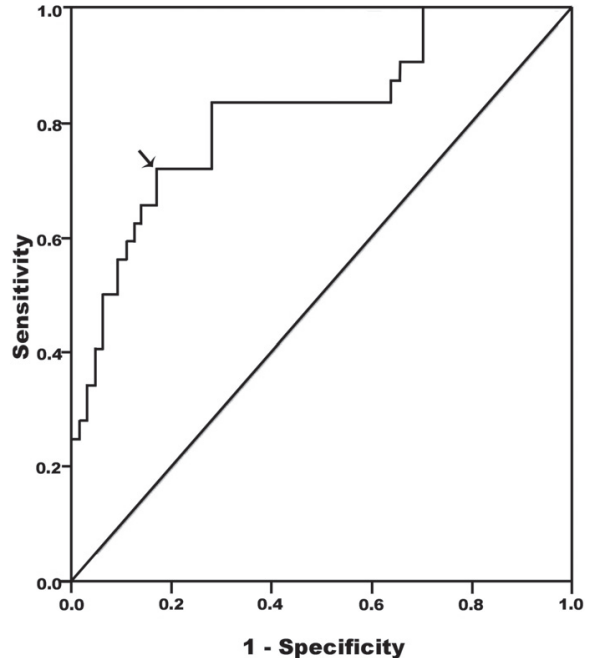

Figure 1. Receiver-operating characteristic curve for SDF-1 (area under the curve $0.776,95 \% \mathrm{CI} 0.735-0.816, P<0.001)$. Arrow indicates the cutoff point $(2,175.1 \mathrm{pg} / \mathrm{mL})$. hibitors or ARB; more use of tirofiban; older age; higher TIMI score; longer delay time; increased level of SDF-1, NT-proB$\mathrm{NP}$, peak troponin I and hs-CRP; and greater incidences of composite endpoint, recurrent MI, death, and advanced HF.

Correlations between plasma SDF-1 and other factors in nonSTEACS: Spearman's correlation analysis showed that SDF1 level in non-STEACS patients was negatively related with age $(r=-0.190, P=0.019)$ and positively related with the levels of NT-proBNP $(r=0.266, P<0.001)$, hs-CRP $(r=0.246$, $P<0.001)$, and peak troponin I $(r=0.241, P<0.001)$, while there was no correlation between plasma SDF-1 and gender, BMI, and eGFR (Table III).

SDF-1 and LV echocardiographic measurements: Echocardiographic parameters were available for 638 subjects $(94.1 \%)$ for the index at admission and 6 months of follow-up. At baseline, LV dimensions were dissimilar in the 2 groups, and EF was not significantly different (Table IV). At 6 months, LVESVI, the primary parameter of LV remodeling, ${ }^{20)}$ was increased by 8.9 (7.9-9.8) and $10.9(10.3-11.8) \mathrm{mL} / \mathrm{m}^{2}$ in the lower SDF-1 and higher SDF-1 groups, respectively (Table IV). The change and percent variation of LVESVI from baseline to 6 months

Table II. Baseline Characteristics of Non-STEACS Patients in Relation to SDF-1 Levels on Admission

\begin{tabular}{|c|c|c|c|c|}
\hline & All Patients & Lower SDF-1 & Higher SDF-1 & $P$ \\
\hline$n$ & 678 & 444 & 234 & \\
\hline Age, years & $68(59-75)$ & $67(57-74)$ & $70(61-77)$ & $<0.001$ \\
\hline Male, $n(\%)$ & $361(53.2)$ & $186(54.9)$ & $175(51.6)$ & 0.397 \\
\hline $\mathrm{BMI}, \mathrm{kg} \cdot \mathrm{m}^{-2}$ & $23.6 \pm 3.1$ & $23.6 \pm 3.3$ & $23.7 \pm 3.0$ & 0.576 \\
\hline Current smoking, $n(\%)$ & $247(36.4)$ & $110(32.4)$ & $137(40.4)$ & 0.031 \\
\hline Hypertension, $n(\%)$ & $365(53.8)$ & $165(48.7)$ & $200(59.0)$ & 0.007 \\
\hline Diabetes mellitus, $n(\%)$ & $139(20.5)$ & $55(16.2)$ & $84(24.8)$ & 0.006 \\
\hline Hypercholesterolemia, $n(\%)$ & $230(33.9)$ & $107(31.6)$ & $123(36.3)$ & 0.194 \\
\hline Prior MI, $n(\%)$ & $58(8.6)$ & $20(5.9)$ & $38(11.2)$ & 0.013 \\
\hline Prior chronic HF, $n(\%)$ & $134(19.8)$ & $38(11.2)$ & $96(28.3)$ & $<0.001$ \\
\hline Prior revascularization, $n(\%)$ & $89(13.1)$ & $35(10.3)$ & $54(15.9)$ & 0.031 \\
\hline ST-depression $\geq 0.1 \mathrm{mV}, n(\%)$ & $244(36.0)$ & $86(25.4)$ & $158(46.6)$ & $<0.001$ \\
\hline Peak troponin I levels, $\mu \mathrm{g} / \mathrm{L}$ & $0.76(0.29-1.63)$ & $0.53(0.01-1.25)$ & $0.99(0.54-1.94)$ & $<0.001$ \\
\hline $\mathrm{eGFR}, \mathrm{mL} \cdot \mathrm{min}^{-1} \cdot 1.73 \mathrm{~m}^{-2}$ surface area & $68.1(51.8-88.3)$ & $68.8(51.3-88.3)$ & $67.9(52.0-88.4)$ & 0.588 \\
\hline Delay time, hours & $74.7(60.4-96.7)$ & $71.9(57.5-89.9)$ & $79.2(61.9-102.5)$ & 0.001 \\
\hline Admission to balloon time, hours & $18.1(10.4-27.6)$ & $18.4(10.7-28.2)$ & $17.9(9.5-27.2)$ & 0.323 \\
\hline Killip class II-IV, $n(\%)$ & $194(28.6)$ & $57(16.8)$ & $137(40.4)$ & $<0.001$ \\
\hline Left main artery disease, $n(\%)$ & $68(10)$ & $24(7.1)$ & $44(13.0)$ & 0.011 \\
\hline Triple vessel disease, $n(\%)$ & $213(31.4)$ & $88(26.0)$ & $125(36.9)$ & 0.002 \\
\hline SDF-1, pg/mL & $2041.8(1845.8-2247.5)$ & $1908.0(1775.6-2034.5)$ & $2338.5(2145.1-2338.5)$ & $<0.001$ \\
\hline NT-proBNP, pmol/L & $615.4(391.8-948.7)$ & $513.3(348.9-747.5)$ & $741.9(452.5-1198.9)$ & $<0.001$ \\
\hline Hs-CRP, mg/L & $2.1(1.5-3.2)$ & $1.8(1.1-2.7)$ & $2.4(1.8-3.6)$ & $<0.001$ \\
\hline \multicolumn{5}{|l|}{ Medication at baseline } \\
\hline ACEI or ARB, $n(\%)$ & $512(75.5)$ & $296(87.3)$ & $216(63.7)$ & $<0.001$ \\
\hline$\beta$-Blocker, $n(\%)$ & 465 (68.6) & $251(74.0)$ & $214(63.1)$ & 0.002 \\
\hline Statin, $n(\%)$ & $630(92.9)$ & $317(93.5)$ & $313(92.3)$ & 0.549 \\
\hline Aspirin, $n(\%)$ & $632(93.2)$ & $318(93.8)$ & $314(92.6)$ & 0.541 \\
\hline Clopidogrel, $n(\%)$ & $588(86.7)$ & $289(85.3)$ & $299(88.2)$ & 0.258 \\
\hline Tirofiban, $n(\%)$ & $180(26.5)$ & $69(20.4)$ & $111(32.7)$ & $<0.001$ \\
\hline \multicolumn{5}{|l|}{ TIMI risk score } \\
\hline $3-4, n(\%)$ & $311(45.9)$ & $182(53.7)$ & $129(38.1)$ & $<0.001$ \\
\hline $5-7, n(\%)$ & $367(54.1)$ & $157(46.3)$ & $210(61.9)$ & $<0.001$ \\
\hline \multicolumn{5}{|l|}{ Endpoints } \\
\hline Composite point & $202(29.8)$ & $30(8.8)$ & $172(50.7)$ & $<0.001$ \\
\hline Death & $61(9.0)$ & $8(2.4)$ & $53(15.6)$ & $<0.001$ \\
\hline Recurrent MI & $27(4.0)$ & $6(1.8)$ & $21(6.2)$ & 0.003 \\
\hline Advanced HF & $114(16.8)$ & $16(4.7)$ & $98(28.9)$ & $<0.001$ \\
\hline
\end{tabular}

MI indicates myocardial infarction; HF, heart failure; Delay time, defined as the time from symptom onset to admission; Admission to balloon time, defined as the time from admission to the first balloon inflation; Hs-CRP, high-sensitivity C-reactive protein; ACEI, angiotensin-converting enzyme inhibitor; ARB, angiotensin receptor blocker; and TIMI, Thrombolysis In Myocardial Infarction. 
Table III. Spearman's Correlations Analysis Between Assessed Biomarkers in Non-STEACS Patients

\begin{tabular}{|c|c|c|c|c|c|c|c|c|}
\hline & Statistics Parameter & Age & Male & BMI & eGFR & NT-proBNP & Hs-CRP & Peak troponin I \\
\hline \multirow[t]{2}{*}{ SDF-1 } & $r_{\mathrm{s}}$ & -0.190 & 0.075 & 0.012 & 0.021 & 0.266 & 0.246 & 0.241 \\
\hline & $P$ & 0.019 & 0.102 & 0.764 & 0.590 & $<0.001$ & $<0.001$ & $<0.001$ \\
\hline
\end{tabular}

$r_{\mathrm{s}}$ indicates correlation coefficient.

Table IV. Left Ventricular Echocardiographic Measurements at Baseline and 6 Months

\begin{tabular}{|c|c|c|c|}
\hline & Lower SDF-1 & Higher SDF-1 & $P$ \\
\hline$n$ & 418 & 220 & \\
\hline \multicolumn{4}{|l|}{ LVESVI, $\mathrm{mL} / \mathrm{m}^{2}$} \\
\hline Baseline & $54.1 \pm 6.1$ & $55.5 \pm 6.4$ & 0.005 \\
\hline 6 Months & $63.2 \pm 6.3$ & $66.5 \pm 6.6$ & $<0.001$ \\
\hline Change & $8.9(7.9-9.8)$ & $10.9(10.3-11.8)$ & $<0.001$ \\
\hline Percent variation (\%) & $16.2(14.5-18.9)$ & $20.2(18.0-22.4)$ & $<0.001$ \\
\hline \multicolumn{4}{|l|}{ LVEDVI, $\mathrm{mL} / \mathrm{m}^{2}$} \\
\hline Baseline & $98.7 \pm 7.9$ & $100.0 \pm 8.0$ & 0.029 \\
\hline 6 Months & $116.4 \pm 8.5$ & $119.6 \pm 8.7$ & $<0.001$ \\
\hline Change & $17.7(16.8-18.4)$ & 19.5 (18.9-20.6) & $<0.001$ \\
\hline Percent variation (\%) & $17.5(16.6-18.9)$ & $19.8(18.7-20.9)$ & $<0.001$ \\
\hline \multicolumn{4}{|l|}{ LVEF, \% } \\
\hline Baseline & $44.4(40.2-49.3)$ & $43.9(39.3-49.0)$ & 0.341 \\
\hline 6 Months & $45.2(41.3-49.5)$ & $43.8(40.0-48.4)$ & 0.004 \\
\hline Change & $0.8(-0.6-1.7)$ & $-0.1(-1.3-1.2)$ & $<0.001$ \\
\hline Percent variation (\%) & $1.7(-1.2-4.5)$ & $-0.3(-2.8-3.0)$ & $<0.001$ \\
\hline \multicolumn{4}{|l|}{ LVMI, $\mathrm{g} / \mathrm{m}^{2}$} \\
\hline Baseline & $147.9 \pm 10.7$ & $150.6 \pm 10.0$ & 0.001 \\
\hline 6 Months & $154.4 \pm 11.5$ & $157.4 \pm 11.4$ & 0.001 \\
\hline Change & $6.7(1.8-10.8)$ & $6.5(2.3-11.2)$ & 0.490 \\
\hline Percent variation $(\%)$ & $4.4(1.2-7.4)$ & $4.4(1.4-7.3)$ & 0.772 \\
\hline
\end{tabular}

Table V. Multivariate Logistic Regression Analysis for Left Ventricular Remodeling After Non-STEACS

\begin{tabular}{|c|c|c|c|c|}
\hline & \multicolumn{2}{|c|}{ Univariate Analysis } & \multicolumn{2}{|c|}{ Multivariate Analysis } \\
\hline & OR $(95 \% \mathrm{CI})$ & $P$ & OR $(95 \% \mathrm{CI})$ & $P$ \\
\hline Age & $1.03(1.02-1.05)$ & $<0.001$ & $1.02(1.00-1.04)$ & 0.087 \\
\hline Diabetes mellitus & $1.48(1.01-2.16)$ & 0.044 & $1.04(0.66-1.66)$ & 0.857 \\
\hline Peak troponin I & $2.29(1.68-3.11)$ & $<0.001$ & $1.89(1.24-2.89)$ & $<0.001$ \\
\hline Use of ACEI or ARB & $0.41(0.29-0.60)$ & $<0.001$ & $0.43(0.27-0.69)$ & 0.001 \\
\hline Use of $\beta$-Blocker & $0.30(0.21-0.42)$ & $<0.001$ & $0.24(0.16-0.37)$ & $<0.001$ \\
\hline SDF-1 $>2175.1 \mathrm{pg} / \mathrm{mL}$ & $3.80(2.77-5.23)$ & $<0.001$ & $2.95(2.02-4.30)$ & $<0.001$ \\
\hline NT-proBNP > 615.4 pmol/L (median) & $6.07(4.35-8.47)$ & $<0.001$ & $4.50(3.05-6.65)$ & $<0.001$ \\
\hline
\end{tabular}

Age, male, BMI, hypertension, diabetes mellitus, prior MI, peak troponin I, use of ACE inhibitor or ARB, use of $\beta$-Blocker, SDF-1, NT-proB$\mathrm{NP}$, and hs-CRP were allowed to enter into the stepwise model.

were significantly different between the lower SDF-1 and higher SDF-1 group $(P<0.001)$. Similar effects were also observed in LVEDVI and LVEF, but not in LVMI.

Univariate predictors of LV remodeling are reported in Table V. When clinical characteristics (as listed under Statistical Analysis above) were entered into a multivariate binary logistic model, SDF-1 (odds ratio (OR), 2.95) and NT-proBNP $(\mathrm{OR}, 4.5)$, together with peak troponin I, nonuse of an ACE inhibitor or $\mathrm{ARB}$, and nonuse of a $\beta$-blocker independently predicted LV remodeling.

SDF-1 and composite primary endpoints (death, MI or HF): Univariate predictors of death, MI or HF are described in Table VI. When clinical characteristics (as listed under Statistical Analysis above) were entered into a multivariate Cox proportional hazards model (Table VI), SDF-1 > 2,175.1 pg/mL (Hazard ratio (HR), 2.45) and NT-proBNP > $615.4 \mathrm{pmol} / \mathrm{L}$ (median) (HR, 3.71), together with diabetes mellitus, Killip class $\geq \mathrm{II}$, left main artery disease, and nonuse of a $\beta$-blocker, $\mathrm{ACEI}$ or ARB, independently predicted the composite primary endpoint. Triple vessel disease was also an independent predictor, although the $P$ value was marginally significant $(P=$ 0.052). Age, male, BMI, current smoking, hypertension, hypercholesterolemia, prior MI, prior CHF, prior revascularization, ST-depression, peak troponin I, eGFR, delay time, admission to balloon time, hs-CRP, use of statin, use of aspirin, use of clopidogrel, and use of tirofiban were not independent predictors.

The areas under the ROC curve for SDF-1 $(0.776 ; 95 \%$ CI, 0.735 to 0.816$)$ and NT-proBNP $(0.817 ; 95 \%$ CI, 0.784 to $0.851)$ were similar. The logistic model combining these 2 markers yielded an area under the curve of 0.862 (95\% CI, 0.834 to $0.891 ; P<0.001)$, which exceeded that of SDF-1 $(P<$ $0.001)$ or NT-proBNP alone $(P=0.0001$; comparison of areas under the receiver operating characteristic curves by the meth- 
Table VI. Multivariate Cox Regression Analysis for Composite Point or Secondary Endpoints after Non-STEACS

\begin{tabular}{|c|c|c|c|c|c|c|c|c|}
\hline & \multicolumn{2}{|c|}{ Composite Point } & \multicolumn{2}{|l|}{ Death } & \multicolumn{2}{|c|}{ Recurrent MI } & \multicolumn{2}{|c|}{ Advanced HF } \\
\hline & $\mathrm{HR}(95 \% \mathrm{CI})$ & $P$ & $\mathrm{HR}(95 \% \mathrm{CI})$ & $P$ & $\mathrm{HR}(95 \% \mathrm{CI})$ & $P$ & $\mathrm{HR}(95 \% \mathrm{CI})$ & $P$ \\
\hline Diabetes mellitus & $1.60(1.17-2.20)$ & 0.004 & & & $4.34(1.84-10.26)$ & 0.001 & & \\
\hline Hypercholesterolemia & & & & & $2.30(0.99-5.32)$ & 0.053 & & \\
\hline Prior revascularization & & & & & $2.94(1.09-7.94)$ & 0.033 & & \\
\hline Peak troponin I & & & & & & & $1.52(0.98-2.34)$ & 0.060 \\
\hline Killip class $\geq$ II & $1.40(1.03-1.89)$ & 0.029 & & & & & $1.64(1.10-2.44)$ & 0.016 \\
\hline Left main artery disease & $1.85(1.26-2.72)$ & 0.002 & & & $3.57(1.39-9.13)$ & 0.008 & $1.76(1.04-2.97)$ & 0.035 \\
\hline Triple vessel disease & $1.35(1.00-1.83)$ & 0.052 & & & & & & \\
\hline SDF-1 > $2175.1 \mathrm{pg} / \mathrm{mL}$ & $2.45(1.71-3.50)$ & $<0.001$ & $3.62(1.79-7.30)$ & $<0.001$ & $2.70(0.94-7.81)$ & 0.046 & $3.15(1.90-5.21)$ & $<0.001$ \\
\hline NT-proBNP > $615.4 \mathrm{pmol} / \mathrm{L}$ (median) & $3.71(2.41-5.70)$ & $<0.001$ & $4.83(2.10-11.06)$ & $<0.001$ & & & $3.85(2.15-6.92)$ & $<0.001$ \\
\hline Use of ACEI or ARB & $0.49(0.34-0.72)$ & $<0.001$ & $0.43(0.21-0.87)$ & 0.019 & $0.34(0.11-1.05)$ & 0.061 & $0.58(0.35-0.96)$ & 0.034 \\
\hline Use of $\beta$-Blocker & $0.52(0.36-0.75)$ & $<0.001$ & & & & & $0.50(0.31-0.81)$ & 0.005 \\
\hline Use of clopidogrel & & & & & $0.28(0.11-0.75)$ & 0.012 & & \\
\hline
\end{tabular}

Age, male, BMI, current smoking, hypertension, diabetes mellitus, hypercholesterolemia, prior MI, prior chronic HF, prior revascularization, ST-depression $\geq 0.1 \mathrm{mV}$, peak troponin I, eGFR, delay time, admission to balloon time, Killip class $\geq$ II, left main artery disease, triple vessel disease, SDF-1, NT-proBNP, Hs-CRP, use of ACEI or ARB, use of $\beta$-Blocker, use of statin, use of aspirin, use of clopidogrel, and use of tirofiban were allowed to enter into the multivariate Cox regression model.

od of Hanley and McNeil). ${ }^{17)}$ When added to the TIMI Risk Score for non-STEACS, the addition of biomarker status to the TIMI Risk Score significantly improved the area under the curve from $0.862(95 \% \mathrm{CI}, 0.834$ to $0.891 ; P<0.001)$ to 0.874 (95\% CI, 0.848 to $0.899 ; P<0.001$ ).

Kaplan-Meier survival curves that plot quartiles of SDF-1 or NT-proBNP (Figure 2A, Figure 2B) provide visual confirmation of the findings from the Cox models, in other words, that both SDF-1 and NT-proBNP are useful predictors of death, MI or HF after non-STEACS (log rank for trend, $P<$ 0.001 ). In patients stratified by NT-proBNP, SDF-1 $>2,175.1$ $\mathrm{pg} / \mathrm{mL}$ provided additional information on death, MI or HF in those patients who had an NT-proBNP level above the median $615.4 \mathrm{pmol} / \mathrm{L}$ (Figure 2C). Thus, patients can be classified into low (SDF-1 $<2,175.1 \mathrm{pg} / \mathrm{mL}$ and NT-proBNP $<615.4 \mathrm{pmol} /$ $\mathrm{L})$, intermediate $(\mathrm{SDF}-1<2,175.1 \mathrm{pg} / \mathrm{mL}$ and NT-proBNP $>$ $615.4 \mathrm{pmol} / \mathrm{L}$, or SDF-1 > 2,175.1 pg/mL and NT-proBNP < $615.4 \mathrm{pmol} / \mathrm{L}$ ), or high risk (SDF-1 $>2,175.1 \mathrm{pg} / \mathrm{mL}$ and NTproBNP $>615.4 \mathrm{pmol} / \mathrm{L})$ groups (log rank for trend, $P<$ 0.001).

SDF-1 and secondary endpoints: SDF-1 was significantly higher in patients who died than in event-free survivors (Table II). Multivariate Cox proportional hazards modeling suggested that the variables (SDF-1, NT-proBNP, nonuse of ACEI or ARB) were independent predictors of death (Table VI). Compared with a lower SDF-1 level, patients with a higher SDF-1 level had a greater incidence of recurrent MI (Table II). Multivariate Cox proportional hazards modeling suggested that SDF-1, diabetes mellitus, prior revascularization, left main artery disease, and nonuse of clopidogrel were independent predictors of recurrent MI (Table VI). We also noticed that hypercholesterolemia and nonuse of an ACEI or ARB resulted in an increased risk for recurrent $\mathrm{MI}$, although the $P$ value was marginally significant $(P=0.053 ; P=0.061$, respectively) (Table VI). SDF-1 levels were also significantly higher in patients who were readmitted with advanced HF than in event-free survivors (Table II). Multivariate Cox proportional hazards modeling revealed the following independent significant predictors: SDF-1, NT-proBNP, Killip class $\geq$ II, left main artery disease, nonuse of an ACE inhibitor or ARB, and nonuse of a $\beta$-blocker (Table VI). Peak troponin I was also an independent predictor, although the $P$ value was marginally significant $(P=0.060)$ (Table VI).

\section{DisCUSSION}

This study demonstrated for the first time that SDF-1 was an independent predictor of left ventricular remodeling and a powerful biomarker of cardiovascular disease and increased risk of death, MI or HF in patients with non-STEACS. The present study was also the first report to compare this with NTproBNP, a well-established biomarker of death and $\mathrm{HF}^{21)}$ Analysis of individual endpoints revealed that SDF-1 contributed to the prediction of death, MI and HF. Consideration of both biomarkers gave added prognostic information above existing clinical characteristics, which enabled patients to be stratified into low-, intermediate-, or high-risk groups.

Our findings suggest that there was an increased level of SDF-1 after non-STEACS. Our data were aligned with other investigations which demonstrated elevated levels of SDF-1 after ST-elevated MI, ${ }^{18,22)}$ non-ST-elevated MI, or unstable angina pectoris. ${ }^{23)}$ It has been reported that HIF-1 induces SDF-1 expression in hypoxic conditions. ${ }^{19)}$ The exact molecular mechanisms of enhanced SDF-1 gene expression in hypoxic conditions are unknown, but hypoxia-induced SDF-1 upregulation in endothelial cells has been ascribed to activation of the transcription factor hypoxia-inducible factor (HIF)-1, which binds to specific binding sites in the SDF-1 promoter in hypoxic conditions thereby inducing SDF-1 expression. ${ }^{19)}$ Interestingly, activation of HIF-1 has been detected in nonhypoxic conditions in vascular SMCs, and nonhypoxic stimuli such as thrombin and platelet-derived growth factor are known to play a relevant role after vascular injury. ${ }^{24,25)}$ These secretory platelet products can increase HIF-1 gene expression and protein translation without being affected by hypoxia. ${ }^{24)}$ This can lead to a substantial accumulation of transcriptionally active HIF-1 in SMCs, which can reach higher levels than after hypoxic stimulation. $^{26)}$

The present study also indicated that non-STEACS patients with increased SDF-1 levels had increased LV dilatation and decreased LVEF during the 6-months of follow-up. Multi- 
A

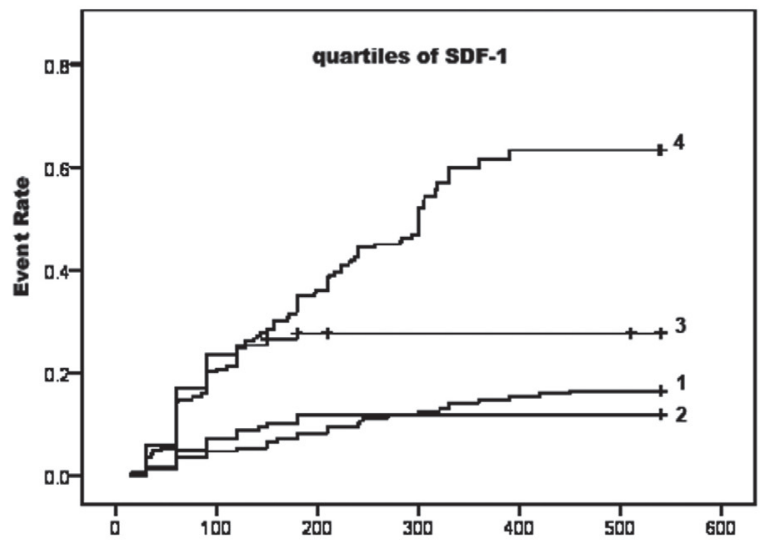

$\mathbf{B}$

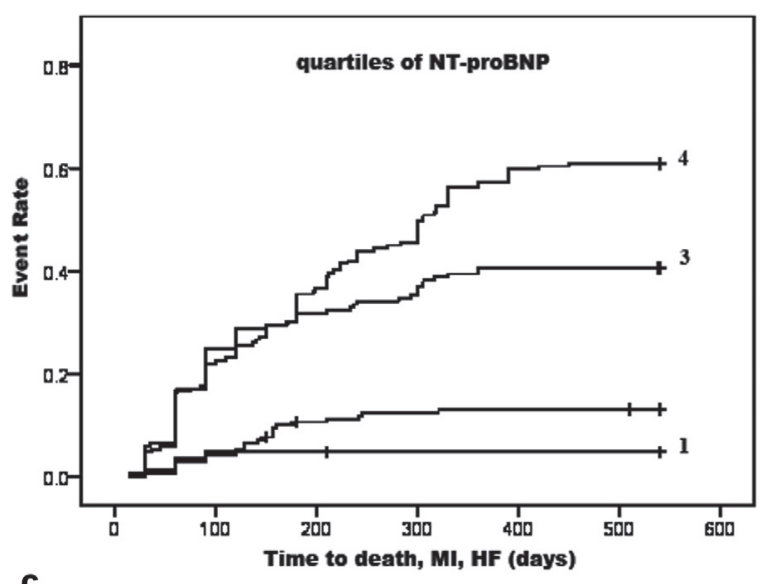

C

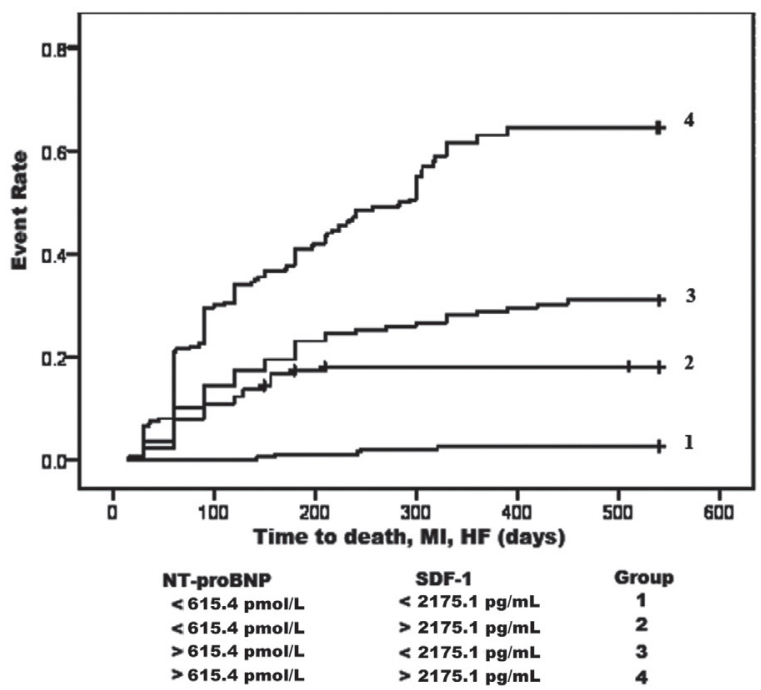

Figure 2. Kaplan-Meier analysis for NT-proBNP, SDF-1 or combination of SDF-1 and NT-proBNP. A: the event rate (death, myocardial infarction (MI) or heart failure (HF)) in patients grouped according to quartiles of plasma SDF-1. B: the event rate (death, myocardial infarction (MI) or heart failure $(\mathrm{HF})$ ) in patients grouped according to quartiles of plasma NT-proBNP. C: This figure shows that plasma SDF-1 levels $(<$ or $>$ $2,175.1 \mathrm{pg} / \mathrm{mL}$ ) predict the primary endpoint of death, myocardial infarction (MI) or heart failure (HF), in patients stratified by NT-proBNP $(<$ or $>$ $615.4 \mathrm{pmol} / \mathrm{L})$. variate logistic regression analysis revealed that SDF-1 independently predicted LV remodeling beyond that provided by established clinical and biochemical markers such as troponin T, NT-proBNP, and hs-CRP. We demonstrated that an elevated SDF-1 level at admission was a significant predictor of death, MI or heart failure through 18 months. Importantly, SDF-1 added to traditional risk factors and provided prognostic information that was independent of and complementary to NTproBNP levels. Risk stratification at an early stage after nonSTEACS remains important and may be useful in helping to select a treatment strategy in the future. A multimarker strategy for outcome management after non-STEACS using independent biomarkers has benefits, because it integrates the different pathways involved, in the hope that complementary information can be gained. ${ }^{27)}$ Receiver operating characteristic curve analysis indicated that NT-proBNP and SDF-1 were of similar accuracy in the prediction of death, MI or HF, but the combination of SDF-1 and NT-proBNP in a multimarker risk stratification approach provided greater predictive accuracy. KaplanMeier analysis revealed that SDF-1 was particularly useful in the group of patients who had elevated NT-proBNP levels (above $615.4 \mathrm{pmol} / \mathrm{L}$ ). In this group, levels of SDF-1 above $2,175.1 \mathrm{pg} / \mathrm{mL}$ were predictive of poor outcome, thus defining a high-risk group. Moreover, adding SDF-1 and NT-proBNP to the TIMI Risk Score for non-STEACS resulted in significantly improved risk stratification and discrimination.

The complementary information provided by SDF-1 to NT-proBNP may suggest that the stimuli to the secretion of both markers are different, and circulating levels are likely to reflect different aspects of cardiovascular homeostasis. The present data showed that SDF-1 independently predicted LV remodeling, and was associated with a higher risk of death, MI or HF. It is well known that myocardial remodeling includes molecular, cellular, and interstitial changes within the myocardium that results in changes in left ventricular size and function. ${ }^{28)}$ One of the predominant features of myocardial remodeling is left ventricular dilatation, ${ }^{29)}$ whose progression affects the survival of the patients with an increased mortality due to heart failure. ${ }^{30)}$

Notably, SDF-1 appears to be actively involved in the pathophysiology of cardiac ischemia-reperfusion injury by promotion of antiapoptotic effects and increase of myocardial STAT3 activation in cardiomyocytes. ${ }^{31)}$ In cultured cardiomyocytes, SDF-1 expression is strongly upregulated by several factors, including GTP cyclohydrolase $\mathrm{I},{ }^{32)}$ heme oxygenase- $1,{ }^{33)}$ hypoxia-inducible factor $1 \mathrm{alpha}^{34)}$ and inflammatory cytokines. ${ }^{35,36)}$ Together, these experimental data suggest that SDF-1 may provide insight into a distinct pathophysiological axis in ACS.

Our study had several limitations. It was a two-center study, and the results need to be replicated in larger multicenter studies. There was a preponderance of non-STEACS, and cutoff points for STEMI may need to be established independently, because the short-term and long-term prognoses of STEMI patients are different from those in non-STEACS patients. ${ }^{37)}$ On the other hand, differences were observed in some of the baseline characteristics in the lower and higher SDF-1 groups. We attempted to minimize the effect of these differences by multivariate analysis. Another limitation is that there was a lack of tissue samples to directly link circulating and tissue concentrations of the biomarker in our study. Plasma levels of 
the biomarker might not be directly related to tissue concentration. Ideally, correlations should have been established between local and systemic biomarkers. Finally, no examinations of SDF-1 level at different times in non-STEACS, including the recovery stage were made presently, so whether convalescent SDF-1 levels have predictive values to the long-term prognosis of non-STEACS remains to be further studied.

Conclusions: The present study is the first report showing SDF-1 to be a new prognostic marker of death, MI or HF in patients with non-STEACS. SDF-1 levels provide prognostic information that is not only independent of traditional risk factors but also complementary to NT-proBNP, and the combination offers improved risk stratification and discrimination compared with the TIMI Risk Score alone. These data demonstrate the prognostic utility of multiple, complementary biomarkers in non-STEACS, and may be useful for risk stratification, and highlight the importance of SDF-1 as a target of further study and potential therapeutic intervention.

\section{ACKNOWLEDGMENTS}

We are indebted to the Catheter Lab technologists at Hangzhou No.1 Municipal Hospital, Nanjing Medical University Affiliated Hangzhou Hospital and Capital Medical University Affiliated Beijing Anzhen Hospital, China.

\section{REFERENCES}

1. Palazzuoli A, Carrera A, Calabria P, et al. Brain natriuretic peptide levels during cardiac reperfusion: comparison between percutaneous coronary angioplasty and aorto-coronaric bypass. Clin Chim Acta 2004; 342: 87-92.

2. Pfeffer MA, Lamas GA, Vaughan DE, Parisi AF, Braunwald E. Effect of captopril on progressive ventricular dilatation after anterior myocardial infarction. N Engl J Med 1988; 319: 80-6.

3. Senior R, Basu S, Kinsey C, Schaeffer S, Lahiri A. Carvedilol prevents remodeling in patients with left ventricular dysfunction after acute myocardial infarction. Am Heart J 1999; 137: 646-52.

4. Farah E, Cogni AL, Minicucci MF, et al. Prevalence and predictors of ventricular remodeling after anterior myocardial infarction in the era of modern medical therapy. Med Sci Monit 2012; 18: CR276-81.

5. Sezer M, Aslanger EK, Cimen AO, et al. Concurrent microvascular and infarct remodeling after successful reperfusion of ST-elevation acute myocardial infarction. Circ Cardiovasc Interv 2010; 3 : 208-15.

6. Richards AM, Doughty R, Nicholls MG, et al. Plasma N-terminal pro-brain natriuretic peptide and adrenomedullin: prognostic utility and prediction of benefit from carvedilol in chronic ischemic left ventricular dysfunction. Australia-New Zealand Heart Failure Group. J Am Coll Cardiol 2001; 37: 1781-7.

7. Jaberg L, Toggweiler S, Puck M, et al. Prognostic value of N-terminal pro-B-type natriuretic peptide in patients with acute coronary syndromes undergoing left main percutaneous coronary intervention. Circ J 2011; 75: 2648-53.

8. Hu X, Dai S, Wu WJ, et al. Stromal cell derived factor-1 alpha confers protection against myocardial ischemia/reperfusion injury: Role of the cardiac stromal cell derived factor- 1 alpha cxcr4 axis. Circulation 2007; 116: 654-63.

9. Elmadbouh I, Haider H, Jiang S, Idris NM, Lu G, Ashraf M. Ex vivo delivered stromal cell-derived factor-1alpha promotes stem cell homing and induces angiomyogenesis in the infarcted myocardium. J Mol Cell Cardiol 2007; 42: 792-803.
10. Saxena A, Fish JE, White MD, et al. Stromal cell-derived factor1alpha is cardioprotective after myocardial infarction. Circulation 2008; 117: 2224-31.

11. Frederick JR, Fitzpatrick JR 3rd, McCormick RC, et al. Stromal cell-derived factor-1alpha activation of tissue-engineered endothelial progenitor cell matrix enhances ventricular function after myocardial infarction by inducing neovasculogenesis. Circulation 2010; 122: S107-17.

12. Choi JO, Kim EY, Lee GY, et al. Predictors of left ventricular reverse remodeling and subsequent outcome in nonischemic dilated cardiomyopathy. Circ J 2013; 77: 462-9.

13. Antman EM, Cohen M, Bernink PJ, et al. The TIMI risk score for unstable angina/non-ST elevation MI: A method for prognostication and therapeutic decision making. JAMA 2000; 284: 835-42.

14. Smilde TD, van Veldhuisen DJ, Navis G, Voors AA, Hillege HL. Drawbacks and prognostic value of formulas estimating renal function in patients with chronic heart failure and systolic dysfunction. Circulation 2006; 114: 1572-80.

15. Lang RM, Bierig M, Devereux RB, et al. Recommendations for chamber quantification: a report from the American Society of Echocardiography's Guidelines and Standards Committee and the Chamber Quantification Writing Group, developed in conjunction with the European Association of Echocardiography, a branch of the European Society of Cardiology. J Am Soc Echocardiogr 2005; 18: 1440-63.

16. Bolognese L, Neskovic AN, Parodi G, et al. Left ventricular remodeling after primary coronary angioplasty: patterns of left ventricular dilation and long-term prognostic implications. Circulation 2002; 106: 2351-7.

17. Hanley JA, McNeil BJ. A method of comparing the areas under receiver operating characteristic curves derived from the same cases. Radiology 1983; 148: 839-43.

18. Chang LT, Yuen CM, Sun CK, et al. Role of stromal cell-derived factor-1alpha, level and value of circulating interleukin-10 and endothelial progenitor cells in patients with acute myocardial infarction undergoing primary coronary angioplasty. Circ J 2009; 73 : 1097-104.

19. Ceradini DJ, Kulkarni AR, Callaghan MJ, et al. Progenitor cell trafficking is regulated by hypoxic gradients through hif-1 induction of sdf-1. Nat Med 2004; 10: 858-64

20. Doughty RN, Whalley GA, Gamble G, MacMahon S, Sharpe N. Left ventricular remodeling with carvedilol in patients with congestive heart failure due to ischemic heart disease. Australia-New Zealand Heart Failure Research Collaborative Group. J Am Coll Cardiol 1997; 29: 1060-6.

21. Wessler BS, Kramer DG, Kelly JL, et al. Drug and device effects on peak oxygen consumption, 6-minute walk distance, and natriuretic peptides as predictors of therapeutic effects on mortality in patients with heart failure and reduced ejection fraction. Circ Heart Fail 2011; 4: 578-88. (Review)

22. Lee BC, Hsu HC, Tseng WY, et al. Effect of cardiac rehabilitation on angiogenic cytokines in postinfarction patients. Heart 2009; 95 : 1012-8.

23. Stellos K, Bigalke B, Langer H, et al. Expression of stromal-cellderived factor- 1 on circulating platelets is increased in patients with acute coronary syndrome and correlates with the number of CD34+ progenitor cells. Eur Heart J 2009; 30: 584-93.

24. Richard DE, Berra E, Pouyssegur J. Nonhypoxic pathway mediates the induction of hypoxia-inducible factor 1alpha in vascular smooth muscle cells. J Biol Chem 2000; 275: 26765-71.

25. Görlach A, Diebold I, Schini-Kerth VB, et al. Thrombin activates the hypoxia-inducible factor-1 signaling pathway in vascular smooth muscle cells: Role of the p22(phox)-containing NADPH oxidase. Circ Res 2001; 89: 47-54.

26. Pagé EL, Robitaille GA, Pouysségur J, Richard DE. Induction of hypoxia-inducible factor-1alpha by transcriptional and translational mechanisms. J Biol Chem 2002; 277: 48403-9.

27. Sabatine MS, Morrow DA, de Lemos JA, et al. Multimarker approach to risk stratification in non-ST elevation acute coronary syndromes: simultaneous assessment of troponin I, C-reactive 
protein, and B-type natriuretic peptide. Circulation 2002; 105: 1760-3.

28. Cohn JN, Ferrari R, Sharpe N. Cardiac remodeling -- concepts and clinical implications: a consensus paper from an international forum on cardiac remodeling. Behalf of an International Forum on Cardiac Remodeling. J Am Coll Cardiol 2000; 35: 569-82. (Review)

29. Cintron G, Johnson G, Francis G, Cobb F, Cohn JN. Prognostic significance of serial changes in left ventricular ejection fraction in patients with congestive heart failure. The V-HeFT VA Cooperative Studies Group. Circulation 1993; 87: VI17-23.

30. White HD, Norris RM, Brown MA, Brandt PW, Whitlock RM, Wild CJ. Left ventricular end-systolic volume as the major determinant of survival after recovery from myocardial infarction. Circulation 1987; 76: 44-51.

31. Huang C, Gu H, Zhang W, Manukyan MC, Shou W, Wang M SDF-1/CXCR4 mediates acute protection of cardiac function through myocardial STAT3 signaling following global ischemia/ reperfusion injury. Am J Physiol Heart Circ Physiol 2011; 301: H1496-505.

32. Ionova IA, Vásquez-Vivar J, Cooley BC, et al. Cardiac myocytespecific overexpression of human GTP cyclohydrolase I protects against acute cardiac allograft rejection. Am J Physiol Heart Circ Physiol 2010; 299: H88-96.

33. Lakkisto P, Kytö V, Forsten H, et al. Heme oxygenase-1 and carbon monoxide promote neovascularization after myocardial infarction by modulating the expression of HIF-1alpha, SDF-1alpha and VEGF-b. Eur J Pharmacol 2010; 635: 156-64.

34. Tillmanns J, Rota M, Hosoda T, et al. Formation of large coronary arteries by cardiac progenitor cells. Proc Natl Acad Sci U S A 2008; 105: 1668-73.

35. Guo WY, Zhang DX, Li WJ, et al. Akt-centered amplification loop plays a critical role in vascular endothelial growth factor/stromal cell-derived factor 1- $\alpha$ cross-talk and cardioprotection. Chin Med J (Engl) 2011; 124: 3800-5.

36. Hohensinner PJ, Kaun C, Rychli K, et al. The inflammatory mediator oncostatin $\mathrm{M}$ induces stromal derived factor-1 in human adult cardiac cells. FASEB J 2009; 23: 774-82.

37. Abbott JD, Ahmed HN, Vlachos HA, Selzer F, Williams DO. Comparison of outcome in patients with ST-elevation versus nonST-elevation acute myocardial infarction treated with percutaneous coronary intervention (from the National Heart, Lung, and Blood Institute Dynamic Registry). Am J Cardiol 2007; 100: 190- 